\title{
A Systematic Review of the Literature on the Relationships between Chronic Diseases and Food Insecurity
}

\author{
Lesley Jo Weaver*, Connor B. Fasel \\ Department of Anthropology, University of Alabama, Tuscaloosa, USA \\ Email: *ljweaver@ua.edu
}

How to cite this paper: Weaver, L.J. and Fasel, C.B. (2018) A Systematic Review of the Literature on the Relationships between Chronic Diseases and Food Insecurity. Food and Nutrition Sciences, 9, 519-541. https://doi.org/10.4236/fns.2018.95040

Received: December 20, 2017

Accepted: May 19, 2018

Published: May 22, 2018

Copyright $\odot 2018$ by authors and Scientific Research Publishing Inc. This work is licensed under the Creative Commons Attribution International License (CC BY 4.0).

http://creativecommons.org/licenses/by/4.0/

\begin{abstract}
Background: The association between food insecurity (FI) and obesity is now so well documented that scholars have dubbed it the "new' food insecurity" and cited it as a leading cause of increased chronic disease (CD) risk. Here, the chain of causality is assumed to be FI $\rightarrow$ obesity $\rightarrow \mathrm{CD}$. However, this model overlooks the possible direct associations between FI and CD independent of obesity. Aim: This study assessed the literature on relationships between FI and CD. Methods: We conducted a systematic literature review of electronic databases. Selection criteria were designed to elicit studies that assessed FI and CD using a measure of CD other than obesity. Results: Fifty-one articles met the inclusion criteria. Forty-five studies (87\%) reported a statistically significant association between FI and CD, but only 15 adjusted for obesity. The association was less consistent for asthma and dyslipidemia than for diabetes, hypertension, and other diet-related CDs, and most were conducted in the USA or Canada. Conclusion: There is a body of literature documenting relationships between FI and CD, but it is heavily biased toward Western nations, ecological study designs, and type 2 diabetes as the $\mathrm{CD}$ of focus. A small subset of the literature controls for BMI, demonstrating that a portion of the FI-CD relationship cannot be fully explained by obesity. Possible direct pathways linking FI and CD include systematic effects of poverty that accompanies FI, micronutrient deficiencies, and environmental exposure to toxins; however, exploration of these alternative pathways is limited by study designs that fail to include obesity as a control variable.
\end{abstract}

\section{Keywords}

Food Insecurity, Chronic Diseases, Systematic Literature Review, Critical Nutrition Studies 


\section{Introduction}

The ecological association between food insecurity (FI) and obesity is now so well documented that scholars have dubbed this pattern the "new' food insecurity" [1] [2] [3] [4] [5] -an especially challenging public health nutrition problem because it appears to be a bimodal, urban phenomenon encompassing "under- and over-consumption, hunger and obesity, quality and quantity" [2]. Based on the growing literature confirming relationships between food insecurity and obesity, many studies make the understandable assumption that there is a greater risk of chronic diet-related diseases among the food insecure, but they often do so without directly measuring that risk [6]. Even the American Dietetic Association (ADA) now considers food insecurity a risk factor for the development of chronic diseases specifically because of the increased risk of obesity among the food insecure, but this approach overlooks the potential health-demoting effects of FI for health independent of obesity [7] [8].

Several recent literature reviews have attempted to elucidate the relationships between various chronic diseases and food insecurity. They evince a strong focus specifically on diet-related chronic diseases, and, following the ADA's assertion that obesity is a risk factor for chronic diseases, emphasize obesity as the primary mediator that links food insecurity with diet-related chronic diseases [9]-[14]. Very few studies have directly assessed this assumed relationship; rather, most uncritically rely on obesity as a proxy for CD risk, as the ADA now does [8].

Obesity is not, however, a necessary precondition for the development of diet-related or non-diet-related chronic diseases, as the literature from India-one of the world's diabetes capitals-has recently demonstrated [15] [16]. Here, the "thin-fat" phenotype, which occurs when fat is added to an already thin body frame with comparatively low muscle mass, increases metabolic disease risk in the absence of clinical obesity or visible overweight. This is most common among adults born at low birth weight who experienced rapid catch-up growth in childhood [15]. More broadly, literature on early-life adversity demonstrates that experiences of famine in utero or early childhood are related to higher risk for cardiometabolic diseases in adulthood, even when controlling for obesity [17] [18] [19] [20] [21].

The characterization in the literature of BMI as a reliable proxy measure for cardiometabolic risk is a problem for two key reasons. First, the flaws of BMI as an indicator of health risk are becoming increasingly clear. BMI is subject to change over the life course, and the physiological risk associated with high BMI can vary quite significantly across populations. Hruschka and Hadley [22], for instance, shows how basal BMI differs dramatically across global populations, leading to the significant misclassification of both under- and overnutrition in many populations. Even among populations for whom BMI works reliably as an indicator of cardiovascular risk, FI-obesity relationship may be inflated when studies rely on self-reported height and weight data, a common practice. Lyons, Park, and Nelson [23] compared self-reported and measured height and weight 
and found that only the former demonstrated an association between FI and body mass index (BMI); when measured height and weight were used, the FI-BMI association disappeared. Despite the problems associated with BMI as a measure of disease risk, the assumption that people with high BMI are essentially people with chronic diseases waiting to happen typically goes unchallenged.

Second, the lack of direct assessment of the relationship between food insecurity and chronic diseases is a problem because it overlooks the possible heightened risk of chronic disease among people suffering from food insecurity who are not obese. It also overlooks the many non-lifestyle-related chronic diseases that are influenced by experiences of food insecurity via stress pathways.

Food insecurity is a complex phenomenon whose negative effects extend well beyond dietary quality and quantity. It is related, for instance, to core aspects of wellbeing including immune function, social relationships, and mental health-all of which may exert their own effects on chronic disease processes independently of their association (or lack thereof) with BMI [12] [24]. Therefore the dominant chain of causality, which is assumed to be food insecurity $\rightarrow$ obesity $\rightarrow$ chronic disease, may be flawed because the link between obesity and chronic diseases is not always straightforward, and because the link between food insecurity and chronic diseases may be either direct or indirect. As a result, the study of alternate pathways from FI to adverse health outcomes is becoming increasingly of interest [25].

This systematic review is designed to answer the question: What is the state of existing knowledge on the pathways between food insecurity and chronic diseases? In other words, it seeks to avoid over-reliance on obesity as the mediator in the FI $\rightarrow$ obesity $\rightarrow \mathrm{CD}$ relationship that is present in much of the literature. Instead it focuses on studies that directly assess, or have the potential to assess, the $\mathrm{FI} \rightarrow \mathrm{CD}$ relationship.

\section{Methods}

\subsection{Literature Search}

The literature search was designed to elicit peer-reviewed articles on the relationships between food insecurity (FI) and chronic diseases (CD), and that employed a measure of CD other than obesity. We conducted a systematic search of Medline, JSTOR, Worldcat, Scopus, Public Affairs Index (PAI), Social Services Abstracts, Annual Reviews Online, and Anthro Source electronic databases. In each database, we conducted a series of searches using an algorithm of all possible combinations of keywords in the following two columns:

$\begin{array}{ll}\text { Column } 1 & \text { Column } 2 \\ \text { Food insecurity } & \text { Chronic disease } \\ \text { Food availability } & \text { Noncommunicable disease } \\ \text { Food insufficiency } & \text { Chronic fatigue } \\ \text { Food shortage } & \text { Fibromyalgia } \\ \text { Food sufficiency } & \text { Lupus }\end{array}$




\section{Obesity \\ Disability \\ Cardiovascular \\ COPD \\ Asthma \\ Hypertension \\ Diabetes \\ Dyslipidemia}

These keywords were obtained from prior examination of the literature. This list of chronic diseases is by no means exhaustive, but they were selected to represent a range of diet-related, environmental, and idiopathic conditions while still remaining manageable in scope. Search results were sorted first by relevance, and second, by recency of publication; the first 200 results of each search were screened. No specific publication dates were selected for limiting the literature search, but sorting search results by recency and considering only the first 200 results of each search confined our results to articles published during or after the year 2000. Studies were retained for the literature review only if they met the following inclusion/exclusion criteria:

1) Study includes a CD assessment beyond general health self-report, and a FI assessment beyond receiving government-sponsored nutritional benefits or living in an area designated as a "food desert."

2) Article reports on the strength of association or the nature of the relationship between FI and CD.

3) Article is a peer-reviewed original study, published in English or with an abstract in English, and deals with humans (not animal models).

4) Reported CD is not skeletal (e.g., arthritis, osteoporosis), not cancer, nor the result of infectious agents (e.g., HIV/AIDS, cervical cancer caused by the $\mathrm{HPV}$ virus). Pre-disease risk states, such as dyslipidemia and insulin resistance, were included.

5) Article does not deal with voluntary food restriction, as in cases of anorexia nervosa, or acute hunger, as in famines.

Potentially relevant articles were downloaded and retained for analysis by the second author; this initial search process resulted in 196 potential articles for inclusion, which were then reviewed by the first author and reduced to 26 articles based on the inclusion and exclusion criteria listed above. To ensure thoroughness, we subsequently performed a cited-reference search ("reverse search") for each article using Google Scholar and reviewed the first 30 results of each search. This resulted in an additional 23 articles fitting the inclusion criteria. Finally, we mined the bibliographies of the retained articles, which led us to an additional 2 meeting the inclusion criteria.

In the qualitative and case-control literature, decisions about the inclusion or exclusion of studies were not always straightforward because assessment of FI or CD was often not quantified or was used as a sample selection criterion rather than a study variable. In such cases, we skimmed results and discussion sections 
and included them when they involved explicit discussion of FI-CD relationships.

\subsection{Data Extraction}

The two authors independently reviewed each article and extracted the following data: study location, design (sample and study type), measure of food insecurity, measure of chronic disease, reported association between the two, control variables used in multivariate analyses (if any), and authors' discussions about the nature of the observed association, or lack thereof. In cases where there were discrepancies in the data extracted from the articles, the first author re-reviewed the article.

\section{Results}

The final number of articles included in the review was 51. A summary of the studies and their attributes is presented in Table 1, while details of each study are presented in Table 2 . They came exclusively from the nutrition, chronic disease, and global health literature.

The 51 studies covered nine countries, with 32 (64 percent) based in the continental U.S., eight (16 percent) in Canada, one (2 percent) in Australia, and the

Table 1. Attributes of the 51 studies included in this literature review.

\begin{tabular}{|c|c|c|c|}
\hline Year of publication & Number (percent) & Study population & Number (percent) \\
\hline $2000-2006$ & $5(10)$ & People with CD & $19(38)$ \\
\hline $2007-2012$ & $21(41)$ & People with FI & $1(2)$ \\
\hline 2013-2015 & $24(47)$ & General or mixed & $31(62)$ \\
\hline Study design & & Chronic disease & \\
\hline Cross-sectional & $42(82)$ & Diabetes & $39(76)$ \\
\hline Longitudinal & $3(6)$ & Hypertension & $12(24)$ \\
\hline \multirow[t]{2}{*}{ Case-control } & $6(12)$ & Dyslipidemia & $10(20)$ \\
\hline & & Asthma & $5(10)$ \\
\hline Study setting & & Cardiovascular disease & $4(8)$ \\
\hline USA & $32(63)$ & Metabolic syndrome & $2(4)$ \\
\hline Canada & $8(16)$ & Kidney disease & $1(2)$ \\
\hline Malaysia & $3(5)$ & & \\
\hline Iran & $2(4)$ & FI Measurement & \\
\hline Mexico & $1(2)$ & HFSSM & $39(76)$ \\
\hline Brazil & $1(2)$ & Other & $12(24)$ \\
\hline Jordan & $1(2)$ & & \\
\hline Kenya & $1(2)$ & Main finding & \\
\hline Puerto Rico & $1(2)$ & Positive association-FI \& CD & $42(82)$ \\
\hline Australia & $1(2)$ & No positive association & $9(18)$ \\
\hline
\end{tabular}


Table 2. Details of the 51 studies included in this literature review.

\begin{tabular}{|c|c|c|c|c|c|c|c|}
\hline Study & $\begin{array}{l}\text { Sample } \\
\text { size }\end{array}$ & $\begin{array}{c}\text { Sample } \\
\text { type and } \\
\text { composition }\end{array}$ & $\begin{array}{l}\text { Location } \\
\text { of study }\end{array}$ & $\begin{array}{l}\text { Study } \\
\text { design }\end{array}$ & $\begin{array}{l}\text { Chronic } \\
\text { disease } \\
\text { measure }\end{array}$ & $\begin{array}{c}\text { Food } \\
\text { insecurity } \\
\text { measure }\end{array}$ & Main result \\
\hline $\begin{array}{l}\text { Asaad and } \\
\text { Chan } 2012\end{array}$ & 16 & $\begin{array}{l}\text { convenience, } \\
\text { elders } 60+\text { with } \\
\text { type } 2 \text { diabetes }\end{array}$ & Canada & Cross-sectional & $\begin{array}{l}\text { dietary quality } \\
\text { and dietary } \\
\text { adherence, } \\
\text { knowledge of } \\
\text { appropriate diet }\end{array}$ & $\begin{array}{l}\text { HFSSM, } \\
\text { slightly } \\
\text { adapted }\end{array}$ & $\begin{array}{l}\text { No significant associations } \\
\text { between FI and CD. }\end{array}$ \\
\hline $\begin{array}{c}\text { Azizi } \\
\text { et al. } 2014\end{array}$ & 260 & $\begin{array}{l}\text { convenience; } \\
\text { all women, } \\
\text { half with MetS }\end{array}$ & Iran & Case-control & $\begin{array}{l}\text { Prior diagnosis } \\
\text { of Metabolic } \\
\text { Syndrome based } \\
\text { on the Adult } \\
\text { Treatment Pane III }\end{array}$ & HFSSM & $\begin{array}{l}\text { FI significantly associated with } \\
\text { metabolic syndrome } \\
(\text { OR 3.2; } 95 \% \text { CI } 1.9-5.6) \\
(P<0.05)\end{array}$ \\
\hline $\begin{array}{c}\text { Bawadi et al. } \\
\quad 2012\end{array}$ & 843 & $\begin{array}{l}\text { convenience; } \\
\text { adults with type } \\
2 \text { diabetes }\end{array}$ & Jordan & Cross-sectional & $\mathrm{HbAlc}$ & $\begin{array}{l}\text { HFSSM, } \\
\text { short form }\end{array}$ & $\begin{array}{l}\text { Moderate and severe FI } \\
\text { associated with poor glycemic } \\
\text { control }(\mathrm{p}=0.04)\end{array}$ \\
\hline $\begin{array}{l}\text { Berkowitz } \\
\text { et al. } 2013\end{array}$ & 2557 & $\begin{array}{l}\text { representative; } \\
\text { adults with type } 1 \\
\text { or } 2 \text { diabetes or } \\
\text { diabetes meication } \\
\text { use from NHANES }\end{array}$ & USA & Cross-sectional & $\mathrm{HbAlc}$ & HFSSM & $\begin{array}{l}\text { FI associated with poor glycemic } \\
\text { control }(\mathrm{p}<0.001) \text { and LDL } \\
\text { control }(\mathrm{p}=0.002) \text { after } \\
\text { controlling for covariates, } \\
\text { but not with BP. }\end{array}$ \\
\hline $\begin{array}{l}\text { Bhargava } \\
\text { et al. } 2012\end{array}$ & 903 & $\begin{array}{l}\text { representative; } \\
\text { elders in Georgia } \\
\text { (no age specified) }\end{array}$ & USA & Cross-sectional & $\begin{array}{l}\text { Comorbidities } \\
\text { numeric count }\end{array}$ & HFSSM & $\begin{array}{l}\text { FI and non-FI adults equally } \\
\text { likely to have Medicare and } \\
\text { out-of-pocket health expenditures } \\
(\mathrm{p}=0.40) \text {, but expenditures are } \\
\text { lower for FI adults }(\mathrm{p}<0.05) \text {. }\end{array}$ \\
\hline $\begin{array}{l}\text { Chan } \\
\text { et al. } 2015\end{array}$ & 21 & $\begin{array}{l}\text { convenience; adults } \\
\text { with diagnosed type } \\
1 \text { or } 2 \text { diabetes who } \\
\text { had experienced FI } \\
\text { in previous year }\end{array}$ & Canada & $\begin{array}{l}\text { qualitative } \\
\text { cross-sectional }\end{array}$ & $\begin{array}{l}\text { Self-reported } \\
\text { diagnosis of type } 1 \\
\text { or } 2 \text { diabetes by a } \\
\text { healthcare } \\
\text { professional }\end{array}$ & $\begin{array}{l}3 \text { question } \\
\text { instrument } \\
\text { derived } \\
\text { from } \\
\text { HFSSM }\end{array}$ & $\begin{array}{l}\text { People with diabetes report } \\
\text { access and preparation barriers } \\
\text { to diabetes-friendly foods and } \\
\text { social isolation, but also resilience } \\
\text { in the face of these challenges. }\end{array}$ \\
\hline $\begin{array}{l}\text { Chaufan, } \\
\text { Davis, and } \\
\text { Constantino } \\
2011\end{array}$ & 21 & $\begin{array}{l}\text { convenience; } \\
\text { Latino } \\
\text { immigrants } \\
\text { in Northern } \\
\text { California }\end{array}$ & USA & $\begin{array}{l}\text { partially } \\
\text { qualitative } \\
\text { cross-sectional }\end{array}$ & Self reported & $\begin{array}{l}\text { Relying on } \\
\text { food aid } \\
\text { programs } \\
(\mathrm{y} / \mathrm{n})\end{array}$ & $\begin{array}{l}\text { Participants identified type } 2 \\
\text { diabetes as the greatest health } \\
\text { problem in the community, but } \\
\text { uniformly suffered from } \\
\text { restricted access to healthy food. }\end{array}$ \\
\hline $\begin{array}{c}\text { Cheng } \\
\text { et al. } 2013\end{array}$ & 1733 & $\begin{array}{l}\text { convenience; } \\
\text { people with } \\
\text { type } 2 \text { diabetes }\end{array}$ & Kenya & Cross-sectional & HbAlc & HFIAS & $\begin{array}{l}\text { High prevalence of FI (68\%) } \\
\text { among patients with diabetes } \\
\text { in a rural, resource-constrained } \\
\text { Western Kenyan setting. }\end{array}$ \\
\hline $\begin{array}{l}\text { Crews } \\
\text { et al. } 2014\end{array}$ & 10,365 & $\begin{array}{l}\text { representative; } \\
\text { lower-income } \\
\text { Americans }\end{array}$ & USA & Cross-sectional & $\begin{array}{l}\text { non-fasting plasma } \\
\text { glucose, systolic } \\
\text { and diastolic BP, } \\
\text { serum and urinary } \\
\text { creatinine, } \\
\text { urine albumin }\end{array}$ & $\begin{array}{l}\text { HFSSM, } \\
\text { slightly } \\
\text { adapted }\end{array}$ & $\begin{array}{l}\text { FI related to chronic kidney } \\
\text { disease in a dose-response man- } \\
\text { ner among people with type } 2 \\
\text { diabetes (OR } 1.67,95 \% \text { CI } \\
1.14-2.45 \text { ) or hypertension } \\
\text { (OR } 1.37,95 \% \text { CI } 1.03-1.82 \text { ). }\end{array}$ \\
\hline $\begin{array}{l}\text { Cuesta-Briand, } \\
\text { Saggers, and } \\
\text { McManus } 2011\end{array}$ & 38 & $\begin{array}{l}\text { convenience; } \\
\text { low-income } \\
\text { Australians } \\
\text { with type } \\
2 \text { diabetes }\end{array}$ & Australia & $\begin{array}{l}\text { qualitative } \\
\text { cross-sectional } \\
\text { analysis }\end{array}$ & $\begin{array}{l}\text { number of diabetes } \\
\text { medications used }\end{array}$ & $\begin{array}{l}\text { Qualitative } \\
\text { complaints } \\
\text { about } \\
\text { dependence } \\
\text { on others } \\
\text { for food and } \\
\text { the high } \\
\text { cost of foods }\end{array}$ & $\begin{array}{l}\text { Low-income earners living with } \\
\text { diabetes faced food security and } \\
\text { reported physical and cost } \\
\text { barriers to following a diabetic } \\
\text { diet, despite knowing that they } \\
\text { should do so. }\end{array}$ \\
\hline
\end{tabular}




\section{Continued}

\begin{tabular}{|c|c|c|c|c|c|c|c|}
\hline $\begin{array}{l}\text { de Cássia } \\
\text { Ribeiro-Silva } \\
\text { et al. } 2014\end{array}$ & 1307 & $\begin{array}{l}\text { convenience; } \\
\text { children } \\
\text { in public school }\end{array}$ & Brazil & Cross-sectional & $\begin{array}{l}\text { frequency of } \\
\text { asthma symptoms } \\
\text { (wheezing) in last } \\
12 \text { months }\end{array}$ & $\begin{array}{l}\text { Brazilian } \\
\text { Food } \\
\text { Insecurity } \\
\text { Scale }\end{array}$ & $\begin{array}{l}\text { Asthma severity associated with } \\
\text { moderate FI (OR 1.71, } \\
95 \% \text { CI } 1.01-2.89 \text { ) and severe } \\
\text { FI (OR } 2.51,95 \% \text { CI } 1.28-4.93 \text { ) } \\
\text { in a dose-response manner. }\end{array}$ \\
\hline $\begin{array}{l}\text { Ding } \\
\text { et al. } 2014\end{array}$ & 6577 & $\begin{array}{l}\text { representative; } \\
\text { general population }\end{array}$ & USA & Cross-sectional & HbAlc & HFSSM & $\begin{array}{l}\text { Marginally food insecure and } \\
\text { food insecure men more likely } \\
\text { to have undiagnosed } \\
\text { pre-diabetes than food secure } \\
\text { men when controlling } \\
\text { for covariates (marginal } \\
\text { FI OR } 1.64,95 \% \text { CI } 1.12 \text { - } 2.38 \text {; } \\
\text { FI OR } 2.12,95 \% \text { CI } 1.28 \text { - } 3.49 \text { ), } \\
\text { but not among women. }\end{array}$ \\
\hline $\begin{array}{l}\text { Fitzgerald } \\
\text { et al. } 2011\end{array}$ & 201 & $\begin{array}{l}\text { convenience; } \\
\text { Latina women }\end{array}$ & USA & Case-control & $\begin{array}{l}\text { self-report of } \\
\text { diagnosis of } \\
\text { diabetes }\end{array}$ & $\begin{array}{l}\text { HFSSM, } \\
\text { short form }\end{array}$ & $\begin{array}{l}\text { Individuals with FI more likely } \\
\text { to have type } 2 \text { diabetes } \\
\text { (OR } 3.33,95 \% \text { CI } 1.34-8.23 \text { ). }\end{array}$ \\
\hline Ford 2013 & 10,455 & representative & USA & Cross-sectional & $\begin{array}{l}\text { HbA1c, CRP, and } \\
\text { cotinine; systolic } \\
\text { BP, total } \\
\text { cholesterol and } \\
\text { HDL/LDL } \\
\text { cholesterol }\end{array}$ & HFSSM & $\begin{array}{l}\text { Severely FI adults had elevated } \\
\mathrm{HbAlc}(\mathrm{p}=0.006), \mathrm{CRP} \\
(\mathrm{p}=0.02) \text {, and cotinine } \\
(\mathrm{p}<0.001) \text {, but not BP or } \\
\text { cholesterol. Severely FI adults } \\
\text { had increased predicted } 10 \text {-year } \\
\text { cardiovascular disease risk } \\
\text { (adjusted prevalence ratio } \\
2.38,95 \% \mathrm{CI} 1.31-4.31) .\end{array}$ \\
\hline $\begin{array}{l}\text { Galesloot } \\
\text { et al. } 2012\end{array}$ & 314 & $\begin{array}{l}\text { convenience; } \\
\text { Canadian adults } \\
\text { with diagnosed } \\
\text { type } 1 \text { or } 2 \text { diabetes }\end{array}$ & Canada & $\begin{array}{l}\text { Partially } \\
\text { qualitative } \\
\text { cross-sectional }\end{array}$ & $\begin{array}{l}\text { previous diagnosis } \\
\text { with diabetes } \\
\text { mellitus and } \\
\text { attending } \\
\text { counseling sessions }\end{array}$ & HFSSM & $\begin{array}{l}\text { Higher rate of FI among } \\
\text { individuals with diabetes in } \\
\text { active care than among a general } \\
\text { population sample }(\mathrm{p}<0.001) \text {. }\end{array}$ \\
\hline $\begin{array}{l}\text { Gucciardi } \\
\text { et al. } 2009\end{array}$ & 2523 & $\begin{array}{l}\text { representative; } \\
\text { Canadians } \\
\text { with diabetes }\end{array}$ & Canada & Cross-sectional & $\begin{array}{l}\text { diabetes } \\
\text { medication use, } \\
\text { plus self-report of } \\
\text { diagnoses from } \\
\text { physicians of: } \\
\text { diabetes, } \\
\text { hypertension, heart } \\
\text { disease, stroke, } \\
\text { and glaucoma }\end{array}$ & HFSSM & $\begin{array}{l}\text { FI more prevalent among } \\
\text { individuals with diabetes } \\
(9.3 \%, \text { OR } 8.2-10.4) \text { versus } \\
\text { without diabetes }(6.8 \%, 95 \% \\
\text { CI } 6.5 \text { - } 7.0) \text {, and associated } \\
\text { with several indices of poor } \\
\text { diabetes management, } \\
\text { mental health problems, } \\
\text { and poor quality of life. }\end{array}$ \\
\hline $\begin{array}{c}\text { Hanson and } \\
\text { Olson } 2012\end{array}$ & 225 & $\begin{array}{l}\text { representative; } \\
\text { rural low-income } \\
\text { adults }\end{array}$ & USA & longitudinal & $\begin{array}{l}\text { being in the upper } \\
\text { quartile of Chronic } \\
\text { Health Conditions } \\
\text { Index }\end{array}$ & HFSSM & $\begin{array}{l}\text { Despite strong knowledge of } \\
\text { community resources and } \\
\text { usage of food aid programs, } \\
\text { FI was common (occurring in } \\
65 \% \text { at some point over the } \\
3 \text {-year study period). Enduring } \\
\text { chronic health problems greatly } \\
\text { increased the risk of persistent } \\
\text { FI (OR } 7.01, p<0.01 \text { ), and } \\
\text { education beyond high school } \\
\text { was the only factor protective } \\
\text { against persistent FI ( } \mathrm{p}<0.05) \text {. }\end{array}$ \\
\hline
\end{tabular}




\section{Continued}

\begin{tabular}{|c|c|c|c|c|c|c|c|}
\hline $\begin{array}{l}\text { Hasan-Ghomi } \\
\text { et al. } 2015\end{array}$ & 400 & $\begin{array}{l}\text { adults with and } \\
\text { without type } \\
2 \text { diabetes }\end{array}$ & Iran & Case-control & $\begin{array}{l}\text { fasting plasma } \\
\text { glucose }\end{array}$ & HFSSM & $\begin{array}{l}\text { No significant differences in FI } \\
\text { risk between diabetic and } \\
\text { nondiabetic groups. Women } \\
\text { were at higher risk of FI } \\
\text { regardless of diabetes status } \\
\text { (nondiabetic OR 3.2, } \\
95 \text { CI } 1.3 \text { - } 7.7 \text {; diabetic OR 2.4, } \\
95 \% \text { CI } 1.02-5.5 \text { ). }\end{array}$ \\
\hline $\begin{array}{l}\text { Heerman } \\
\text { et al. } 2016\end{array}$ & 401 & $\begin{array}{l}\text { representative; } \\
\text { adults with } \\
\text { diabetes }\end{array}$ & USA & Cross-sectional & $\mathrm{HbAlc}$ & $\begin{array}{l}3 \text { question } \\
\text { instrument } \\
\text { derived from } \\
\text { HFSSM }\end{array}$ & $\begin{array}{l}\text { FI is common in low-SES people } \\
\text { with type } 2 \text { diabetes }(73 \%) \text {; FI } \\
\text { associated with lower adherence } \\
\text { to self-care recommendations } \\
(\mathrm{p}=0.002 \text { - } 0.03 \text { for various } \\
\text { recommendations) and worse } \\
\text { glycemic control }(\mathrm{p}=0.03) .\end{array}$ \\
\hline $\begin{array}{l}\text { Hendrickson } \\
\text { et al. } 2010\end{array}$ & 127 & $\begin{array}{l}\text { convenience; } \\
\text { families presenting } \\
\text { to a hospital for } \\
\text { child asthma }\end{array}$ & USA & Cross-sectional & $\begin{array}{l}\text { frequency of } \\
\text { coughing or } \\
\text { wheezing, night } \\
\text { cough, activity } \\
\text { limitation, missed } \\
\text { school days, return } \\
\text { to ED, length of } \\
\text { hospitalization }\end{array}$ & HFSSM & $\begin{array}{l}\text { FI is common in families of } \\
\text { asthmatic children (35\%), } \\
\text { but is not associated with } \\
\text { indicators of asthma severity. }\end{array}$ \\
\hline $\begin{array}{l}\text { Holben and } \\
\text { Pheley } 2006\end{array}$ & 808 & $\begin{array}{l}\text { convenience; } \\
\text { rural adults }\end{array}$ & USA & Cross-sectional & $\begin{array}{l}\text { diastolic BP, total } \\
\text { cholesterol, } \\
\text { random blood } \\
\text { glucose, HbAlc, } \\
\text { hemoglobin }\end{array}$ & HFSSM & $\begin{array}{l}\text { BMI }(\mathrm{p}=0.04) \text { and obesity } \\
(\mathrm{p}<0.001) \text { were higher among } \\
\text { people with FI, but diastolic BP, } \\
\text { total cholesterol, random blood } \\
\text { glucose, HbAlc, and } \\
\text { hemoglobin did not differ. }\end{array}$ \\
\hline $\begin{array}{l}\text { Homenko } \\
\text { et al. } 2010\end{array}$ & 74 & $\begin{array}{l}\text { representative; } \\
\text { rural older adults } \\
\text { with type } 2 \text { diabetes } \\
\text { (age not specified) }\end{array}$ & USA & Cross-sectional & $\mathrm{HbAlc}$ & $\begin{array}{l}\text { HFSSM, } \\
\text { short form }\end{array}$ & $\begin{array}{l}\text { FI associated with higher BMI } \\
(\mathrm{p}=0.01) \text { but not HbAlc. }\end{array}$ \\
\hline $\begin{array}{l}\text { Hwang and } \\
\text { Bugeja } 2000\end{array}$ & 50 & $\begin{array}{l}\text { convenience; } \\
\text { homeless in } \\
\text { Toronto }\end{array}$ & Canada & $\begin{array}{l}\text { partially } \\
\text { qualitative, } \\
\text { cross-sectional }\end{array}$ & HbAlc & $\begin{array}{l}\text { "difficulties } \\
\text { obtaining the } \\
\text { necessities of } \\
\text { life" scale } \\
\text { derived from } \\
\text { qualitative } \\
\text { interviews }\end{array}$ & $\begin{array}{l}\text { Homeless adults with diabetes } \\
\text { reported difficulties managing } \\
\text { their disease, and poor } \\
\text { glycemic control was common } \\
(44 \%) \text {. However, people rarely } \\
\text { said that poor glycemic } \\
\text { control created difficulties } \\
\text { obtaining the necessities of life. }\end{array}$ \\
\hline $\begin{array}{l}\text { Kollannoor- } \\
\text { Samuel } \\
\text { et al. } 2012\end{array}$ & 211 & $\begin{array}{l}\text { convenience; } \\
\text { Latinos in US with } \\
\text { type } 2 \text { diabetes }\end{array}$ & USA & $\begin{array}{l}\text { Partially } \\
\text { qualitative } \\
\text { cross-sectional }\end{array}$ & $\begin{array}{l}\text { fasting blood } \\
\text { glucose, } \mathrm{HbAlc}\end{array}$ & HFSSM & $\begin{array}{l}\text { FI associated with experiencing } \\
\text { barriers to diabetes self-care } \\
\text { (ORs ranging } 1.22-1.46 \text {, } \\
\text { 95\% CIs ranging } 1.04-1.82 \text { ) }\end{array}$ \\
\hline $\begin{array}{c}\text { Liu } \\
\text { et al. } 2015\end{array}$ & 5533 & $\begin{array}{l}\text { representative; } \\
\text { adults without } \\
\text { diabetes }\end{array}$ & USA & Cross-sectional & $\begin{array}{l}\text { fasting plasma } \\
\text { insulin and } \\
\text { glucose }\end{array}$ & HFSSM & $\begin{array}{l}\text { FI related to insulin resistance } \\
\text { among normal weightmen after } \\
\text { controlling for covariates } \\
\text { (OR } 3.99,95 \% \text { CI } 1.71-9.33 \text { ). } \\
\text { Among women, association was } \\
\text { significant in bivariate analysis } \\
\text { only ( }<<0.001 \text { overweight; } \\
p=0.001 \text { normal weight). }\end{array}$ \\
\hline
\end{tabular}




\section{Continued}

\begin{tabular}{|c|c|c|c|c|c|c|c|}
\hline $\begin{array}{l}\text { Lyles } \\
\text { et al. } 2013\end{array}$ & 665 & $\begin{array}{l}\text { representative; } \\
\text { low-income adults } \\
\text { with type } 2 \text { diabetes }\end{array}$ & USA & longitudinal & HbAlc & HFSSM & $\begin{array}{l}\text { FI associated with higher HbAlc, } \\
\text { lower self-efficacy, and lower } \\
\text { consumption of fruits and veg } \\
\text { (all p < 0.05); but after an } \\
\text { educational intervention, } \\
\text { these differences disappeared. }\end{array}$ \\
\hline $\begin{array}{l}\text { Mangini } \\
\text { et al. } 2015\end{array}$ & 11,099 & $\begin{array}{l}\text { representative; } \\
\text { American } \\
\text { third-graders }\end{array}$ & USA & Cross-sectional & $\begin{array}{l}\text { parental report } \\
\text { of child's } \\
\text { asthma diagnosis }\end{array}$ & HFSSM & $\begin{array}{l}\text { Household FI associated with } \\
\text { child asthma regardless of } \\
\text { race/ethnicity (OR 1.04; } \\
95 \% \text { CI } 1.02 \text { - 1.06), but to } \\
\text { different degrees } \\
\text { between ethnic groups. }\end{array}$ \\
\hline $\begin{array}{l}\text { Marjerrison } \\
\text { et al. } 2011\end{array}$ & 183 & $\begin{array}{l}\text { convenience; } \\
\text { families of children } \\
\text { with type } 1 \text { diabetes }\end{array}$ & Canada & $\begin{array}{l}\text { Partially qualit- } \\
\text { ative } \\
\text { cross-sectional }\end{array}$ & $\mathrm{HbAlc}$ & HFSSM & $\begin{array}{l}\text { Children from FI households } \\
\text { have higher HbA1c }(\mathrm{p}=0.039) \\
\text { and more frequent } \\
\text { hospitalizations }(\mathrm{p}=0.002) \\
\text { but only in bivariate analyses. }\end{array}$ \\
\hline $\begin{array}{l}\text { Mayer } \\
\text { et al. } 2016\end{array}$ & 407 & $\begin{array}{l}\text { convenience; adults } \\
\text { on Medicaid or } \\
\text { living in a zip code } \\
\text { with high poverty, } \\
\text { with diabetes }\end{array}$ & USA & Cross-sectional & HbAlc & HFSSM & $\begin{array}{l}\text { FI associated with poor glucose } \\
\text { control (OR } 2.23,95 \% \\
\text { CI } 1.22-4.1 \text { ), while SNAP } \\
\text { enrollment was associated with } \\
\text { lower risk of poor glucose control } \\
\text { (OR } 0.27,95 \% \text { CI } 0.09-0.08) \text {. }\end{array}$ \\
\hline $\begin{array}{l}\text { Mohamadpour, } \\
\text { Sharif, and } \\
\text { Keysami } 2012\end{array}$ & 169 & $\begin{array}{l}\text { representative; } \\
\text { Malaysian Indian } \\
\text { women }\end{array}$ & Malaysia & Cross-sectional & $\begin{array}{l}\text { BP, lipids, } \\
\text { plasma glucose }\end{array}$ & $\begin{array}{l}\text { Radimer/ } \\
\text { Cornell } \\
\text { Hunger and } \\
\text { Food } \\
\text { Insecurity } \\
\text { Instrument }\end{array}$ & $\begin{array}{l}\text { No significant differences in total } \\
\text { cholesterol, triglycerides, HDL, } \\
\text { LDL, plasma glucose, or blood } \\
\text { pressure by FI status. }\end{array}$ \\
\hline $\begin{array}{l}\text { Moreno } \\
\text { et al. } 2015\end{array}$ & 250 & $\begin{array}{l}\text { convenience; } \\
\text { Latinos } \\
\text { with diabetes }\end{array}$ & USA & Cross-sectional & $\begin{array}{l}\text { LDL cholesterol, } \\
\text { BP, HbAlc }\end{array}$ & HFSSM & $\begin{array}{l}\text { People with type } 2 \text { diabetes and } \\
\text { FI reported more medication } \\
\text { underuse (OR } 2.49,95 \% \mathrm{CI} \\
1.30-4.98 ; \mathrm{p}=0.003) \text {, poorer } \\
\text { diabetes control (OR } 0.24 ; 95 \% \mathrm{CI} \\
0.07-0.84 ; \mathrm{p}<0.05) \text {, and fewer } \\
\text { preventative exams (both } \\
\mathrm{p}<0.05) \text { than those without FI. }\end{array}$ \\
\hline $\begin{array}{l}\text { Nelson } \\
\text { et al. } 2001\end{array}$ & 1503 & $\begin{array}{l}\text { representative; } \\
\text { adults with } \\
\text { diabetes }\end{array}$ & USA & Cross-sectional & $\begin{array}{l}\text { self-reported health } \\
\text { status, number of } \\
\text { physician } \\
\text { encounters, and } \\
\text { number of } \\
\text { hospitalizations }\end{array}$ & HFSSM & $\begin{array}{l}\text { FI was associated with fair or } \\
\text { poor self-reported health status } \\
\text { in bivariate analysis }(\mathrm{p}=0.05) \text { but } \\
\text { not multivariate. In both bivariate } \\
\text { and multivariate analysis, FI } \\
\text { diabetics reported higher } \\
\text { healthcare utilization }(\mathrm{p}=0.05) .\end{array}$ \\
\hline $\begin{array}{l}\text { Nur Atiqah } \\
\text { et al. } 2015\end{array}$ & 124 & $\begin{array}{l}\text { convenience; } \\
\text { young adults } \\
\text { at university }\end{array}$ & Malaysia & Cross-sectional & $\begin{array}{l}\text { Lipid profile, } \\
\text { CRP, and body } \\
\text { composition }\end{array}$ & $\begin{array}{l}\text { Adult Food } \\
\text { Security } \\
\text { Survey } \\
\text { Module }\end{array}$ & $\begin{array}{l}\text { No significant associations } \\
\text { between FI and CD. }\end{array}$ \\
\hline $\begin{array}{l}\text { Parker } \\
\text { et al. } 2010\end{array}$ & 9251 & $\begin{array}{l}\text { representative; } \\
\text { general population }\end{array}$ & USA & Cross-sectional & $\begin{array}{l}\text { total cholesterol, } \\
\text { HDL cholesterol, } \\
\text { BP, blood glucose, } \\
\text { BMI }\end{array}$ & HFSSM & $\begin{array}{l}\text { Adults with marginal or severe } \\
\text { FI more likely to have MetS } \\
\text { (marginal OR } 1.80,95 \% \text { CI } \\
1.30-2.49 \text {, severe OR } 1.65 \text {, } \\
95 \% \text { CI } 1.12-2.42 \text { ), } \\
\text { but not adolescents. }\end{array}$ \\
\hline
\end{tabular}




\section{Continued}

\begin{tabular}{|c|c|c|c|c|c|c|c|}
\hline $\begin{array}{l}\text { Pérez-Escamilla } \\
\text { et al. } 2014\end{array}$ & 32320 & $\begin{array}{l}\text { representative; } \\
\text { general population }\end{array}$ & Mexico & Case-control & $\begin{array}{l}\text { self-reported } \\
\text { doctor diagnoses } \\
\text { of diabetes or } \\
\text { hypertension }\end{array}$ & $\begin{array}{l}\text { Modified } \\
\text { version of } \\
\text { the Latin } \\
\text { American } \\
\text { and } \\
\text { Caribbean } \\
\text { Food } \\
\text { Security } \\
\text { Scale }\end{array}$ & $\begin{array}{l}\text { FI was a risk factor for type } 2 \\
\text { diabetes among women } \\
(p=0.005) \text { but not men } \\
(p=0.349) \text {, and for hypertension } \\
\text { across genders } \\
\text { (women } p=0.037 \\
\text { men } p=0.072)\end{array}$ \\
\hline $\begin{array}{c}\text { Sattler and } \\
\text { Bhargava } 2016\end{array}$ & 2944 & $\begin{array}{l}\text { representative; } \\
\text { adolescents only }\end{array}$ & USA & Cross-sectional & $\begin{array}{l}\text { fasting plasma } \\
\text { glucose and } 2 \text { hour } \\
\text { plasma glucose } \\
\text { following an } \\
\text { OGTT, total } \\
\text { cholesterol, } \\
\text { BP, HbA1c }\end{array}$ & HFSSM & $\begin{array}{l}\text { FI not associated with glucose, } \\
\text { cholesterol, BP, or HbAlc } \\
\text { in bivariate analyses. }\end{array}$ \\
\hline $\begin{array}{c}\text { Sattler and Lee } \\
2013\end{array}$ & 664 & $\begin{array}{l}\text { convenience; older } \\
\text { Americans enrolled } \\
\text { or waitlisted for } \\
\text { Older Americans } \\
\text { Act Nutrition } \\
\text { Program }\end{array}$ & USA & longitudinal & $\begin{array}{l}\text { Blood glucose, } \\
\text { total cholesterol, } \\
\text { BP, healthy diet, } \\
\text { BMI, smoking, } \\
\text { and physical } \\
\text { activity }\end{array}$ & HFSSM & $\begin{array}{l}\text { People with persistent FI over } \\
\text { the } 8 \text {-month period were more } \\
\text { likely to report being diagnosed } \\
\text { with coronary heart disease } \\
\text { and diabetes, and to practice } \\
\text { cost-related medication } \\
\text { nonadherence (all } \mathrm{p}<0.05 \text { ). }\end{array}$ \\
\hline $\begin{array}{l}\text { Seligman } \\
\text { et al. } 2007\end{array}$ & 4423 & $\begin{array}{l}\text { representative; } \\
\text { general population }\end{array}$ & USA & Cross-sectional & $\begin{array}{l}\text { self report and } \\
\text { fasting serum } \\
\text { glucose }\end{array}$ & HFSSM & $\begin{array}{l}\text { Diabetes prevalence was higher } \\
\text { in severely FI groups after } \\
\text { adjusting for sociodemographics, } \\
\text { physical activity level, and BMI } \\
\text { (OR 2.1, 95\% CI } 1.1-4.0, \mathrm{p}=0.02 \text { ). }\end{array}$ \\
\hline $\begin{array}{l}\text { Seligman } \\
\text { et al. } 2010\end{array}$ & 40 & $\begin{array}{l}\text { convenience; } \\
\text { low-income adults } \\
\text { with type } 2 \text { diabetes }\end{array}$ & USA & Cross-sectional & HbAlc & $\begin{array}{l}\text { HFSSM, } \\
\text { short form }\end{array}$ & $\begin{array}{l}\text { FI was associated with inadequate } \\
\text { diabetes control (OR } 1.35,95 \% \text { CI } \\
1.05-1.7 \text { ), and average HbAlc } \\
\text { was higher, though } \\
\text { not statistically significant. }\end{array}$ \\
\hline $\begin{array}{l}\text { Seligman } \\
\text { et al. } 2012\end{array}$ & 711 & $\begin{array}{l}\text { convenience; people } \\
\text { with type } 2 \text { diabetes } \\
\text { in safety net health } \\
\text { clinics }\end{array}$ & USA & Cross-sectional & $\mathrm{HbAlc}$ & HFSSM & $\begin{array}{l}\text { FI is an independent risk factor } \\
\text { for poor glycemic control } \\
\text { (OR } 1.48,95 \% \text { CI } 1.07-2.04 \text { ), } \\
\text { possibly through the mechanisms } \\
\text { of difficulty following a diabetic } \\
\text { diet, and emotional distress } \\
\text { stemming from low diabetes } \\
\text { self-efficacy. }\end{array}$ \\
\hline $\begin{array}{c}\text { Shariff } \\
\text { et al. } 2014\end{array}$ & 625 & $\begin{array}{l}\text { representative; } \\
\text { women only }\end{array}$ & Malaysia & Cross-sectional & $\begin{array}{l}\text { fasting serum } \\
\text { glucose, lipid } \\
\text { profile, BP }\end{array}$ & $\begin{array}{l}\text { Radimer/ } \\
\text { Cornell } \\
\text { Hunger and } \\
\text { Food } \\
\text { Insecurity } \\
\text { Instrument }\end{array}$ & $\begin{array}{l}\text { Women in FI households were } \\
\text { less likely to have elevated } \\
\text { glucose, high total cholesterol } \\
\text { or LDL (all p < } 0.05 \text { ), when } \\
\text { controlling for } \\
\text { sociodemographic covariates. }\end{array}$ \\
\hline Sharkey 2003 & 279 & $\begin{array}{l}\text { representative; } \\
\text { homebound older } \\
\text { women receiving } \\
\text { meal delivery } \\
\text { services }\end{array}$ & USA & Case-control & $\begin{array}{l}\text { self-reported } \\
\text { doctor diagnosis of } \\
9 \text { diseases, plus } \\
\text { effect of disease(s) } \\
\text { on daily activities }\end{array}$ & $\begin{array}{l}4 \text { question } \\
\text { instrument } \\
\text { derived from } \\
\text { elements of } \\
\text { food } \\
\text { insecurity } \\
\text { reported } \\
\text { among elders } \\
\text { in a previous } \\
\text { study }\end{array}$ & $\begin{array}{l}\text { Women with FI were more } \\
\text { likely to report multimorbidity } \\
\text { than those without FI (OR } 3.69 \text {, } \\
95 \% \text { CI } 1.14-12.0, \mathrm{p}<0.001) \text {. }\end{array}$ \\
\hline
\end{tabular}




\section{Continued}

$\begin{array}{ccl}\begin{array}{c}\text { Shin } \\ \text { et al. } 2015\end{array} 1663 & \begin{array}{l}\text { representative; } \\ \text { adults }\end{array}\end{array}$

Shiue 2016

representative; general population

convenience; US

Silverman

et al. 2015

adults with poorly controlled type 2 diabetes

Tarasuk

et al. 2013

representative; general population

Tayie and

Zizza 2009

5549

representative; general population

Terrell, Drew, and Vargas 2009
2 question instrument derived serum lipid levels: from the USA Cross-sectional total cholesterol, Behavioral LDL, and HDL Risk Factor Surveillance System and NHANES

$\mathrm{BP}$, serum glucose,

USA Cross-sectional total cholesterol

HFSSM and HDL, CRP

USA Cross-sectional HbAlc

HFSSM

self report of

Canada Case-control

physician diagnosis HFSSM of 10 conditions

serum fasting triglyceride, total

USA Cross-sectional cholesterol, LDL, HDL, and their ratios

HFSSM

FI associated with prior asthma $(\mathrm{p}=0.002)$, arthritis $(\mathrm{p}=0.051)$, chronic bronchitis $(\mathrm{p}=0.001)$, depression $(\mathrm{p}<0.001)$, diabetes $(p=0.042)$, eczema $(p=0.001)$, emphysema $(p=0.013)$, liver problems $(p=0.022)$, suggesting disability from illness might prevent these individuals from being employed.

FI associated with depression $(\mathrm{p}<0.001)$, diabetes distress $(\mathrm{p}<0.001)$, low medication adherence $(\mathrm{p}=0.02)$, and worse glycemic control $(\mathrm{p}=0.02)$.

Most conditions increased the odds of FI when controlling for other sociodemographics, and this effect became greater with the increasing number of comorbid chronic conditions: OR $1.43,95 \%$ CI 1.28 - 1.59 for one condition, OR 1.86, 95\% CI 1.62 - 2.14 for two, and OR 3.44, 95\% CI 3.02 - 3.93) for 3 or more.

FI not associated with lipid measures among men. Among women, those with moderate food insecurity were more likely to have abnormal LDL ( $p=0.045)$, and those with FI but not hunger had higher abnormal fasting serum triglyceride $(\mathrm{p}=0.041)$.

People with FI were more likely to have diabetes based on self-report (OR 1.45, 95\% CI 1.13 - 1.85). People with FI and hypertension were more likely to have better blood pressure control (OR 1.335, 95\% CI 1.06 - 1.68). 


\section{Continued}

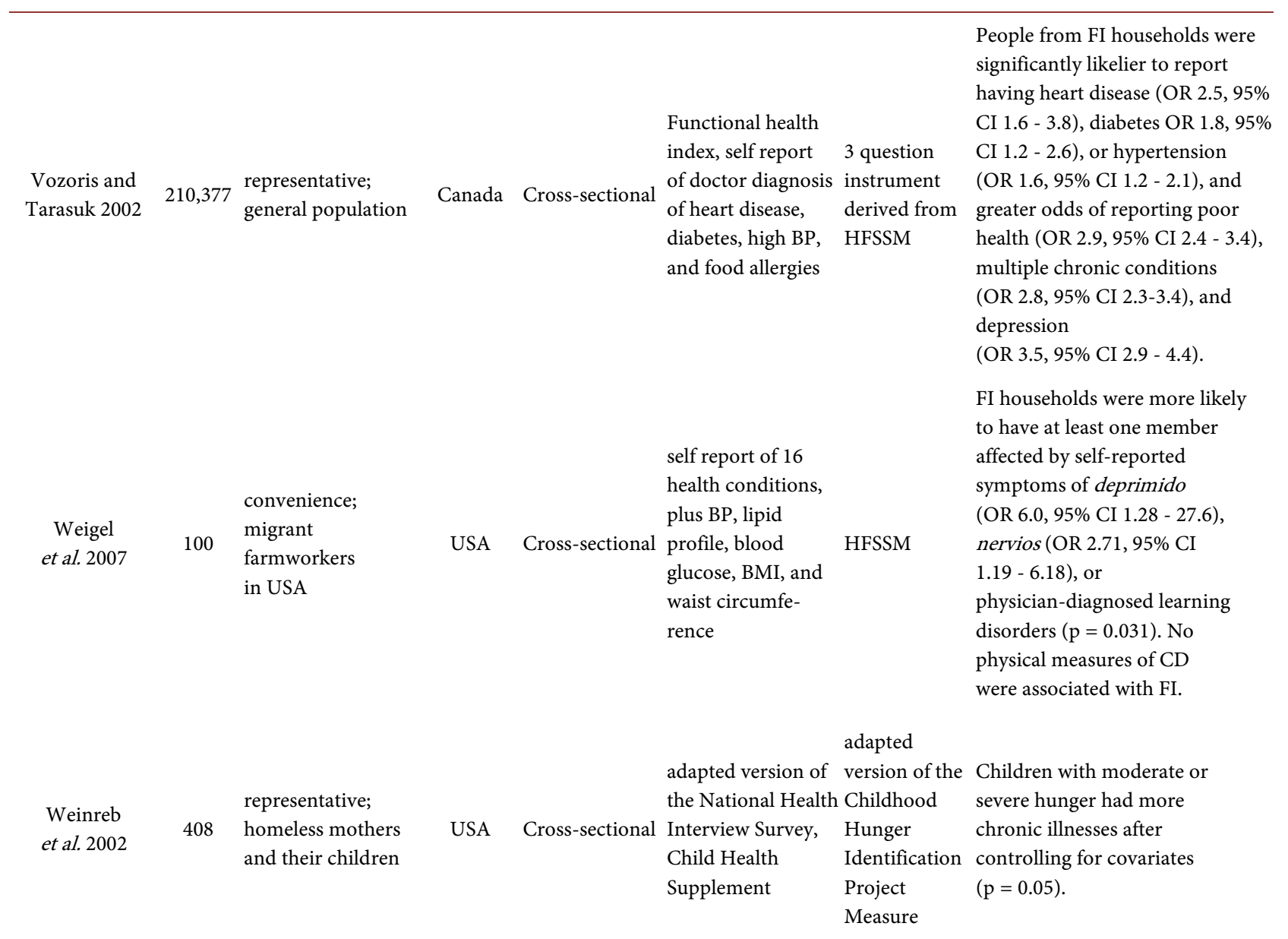

remaining 9 (18 percent) in the following lower- and middle-income countries: Puerto Rico, Malaysia, Iran, Jordan, Kenya, Mexico and Brazil. Among the 9 studies reporting no association between FI and CD were all 3 studies conducted in Malaysia, suggesting that there may not be a FI-CD relationship in that particular locale as compared to the other 8 countries represented in the sample [26] [27] [28].

Sampling and study design varied widely. The vast majority of studies were quantitative ( 45 studies; 90 percent) and cross-sectional (43 studies; 86 percent) in their design. Six studies (12 percent) were partially or entirely qualitative, 3 studies (5 percent) were longitudinal, and 5 studies (10 percent) were case-control studies. The average sample size was 8,397 (range 16 - 210,377). Twenty-three studies ( 46 percent) used convenience samples, while 28 (56 percent) used representative samples, many of which were drawn from large national data sets such as the USA's National Health and Nutrition Examination Survey (NHANES). Nineteen studies (38 percent) recruited participants with CDs already diagnosed, while only 1 study (2 percent) examined CD outcomes among people already identified as FI. The remaining 31 studies (62 percent) examined associations between FI and CD in general samples or those selected 
on a demographic indicator, such as being low-income or being of a specific race or ethnicity. Six studies (12 percent) used women-only samples, and no studies used men-only samples. Minority race/ethnicity and older age were two other common demographic factors on which samples were selected, presumably because these are considered risk factors for FI and/or CD.

The studies largely reported positive relationships between FI and CD. That is, most (42 studies; 82 percent) reported that as FI increased, so did CD prevalence or severity, or the reverse: as CD severity increased, so did FI. The remaining 9 studies (18 percent) reported no positive association between FI and CD [26]-[34].

The strength and consistency of relationships between FI and CD varied depending on the $\mathrm{CD}$ under consideration. The studies reporting on type 1 or 2 diabetes, hypertension, heart disease, the Metabolic Syndrome, and chronic kidney disease found positive associations between FI and CD. There was marked inconsistency, however, in studies dealing with asthma and dyslipidemia. Five of the 10 studies dealing with dyslipidemia reported either no positive relationship, or a positive relationship between FI and dyslipidemia on only some (not all) indicators or in only some portions of their study samples. For instance, Tayie and Zizza's [35] study of a representative sample of US adults reported an association between FI and dyslipidemia, but only for some levels of FI, only among women, and only on some markers of dyslipidemia and not others. Also somewhat inconsistent was the relationship between FI and asthma. While five studies dealt with asthma, only three reported measures of asthma severity, and one of those three found no association between household FI and any of their asthma severity indicators [30].

Only 15 of the total of 51 studies addressing FI-CD relationships controlled for BMI, waist circumference, or another measure of obesity in their multivariate analyses [36]-[50]. With one exception [41], those that did so uniformly documented an association between FI and the CD of interest-which in these cases were type 2 diabetes, asthma, and dyslipidemia-even when controlling for BMI.

The studies reported some differences in the FI-CD relationship along demographic lines. Lower socioeconomic status was the most common predictor of a positive relationship between FI and CD, and many of the studies specifically worked with low-income populations. Minority race/ethnicity, older age, and female gender were also significant demographic predictors of FI-CD relationships, but, as noted above, many of the studies that addressed these variables selected their samples on one or more of these characteristics and therefore lacked a comparison group.

\section{Discussion}

The 51 studies included in this literature review confirm a positive relationship between food insecurity (FI) and chronic diseases (CD), and they suggest some possible mechanisms explaining that association. However, only 15 studies controlled for body mass index (BMI) in their statistical analyses, a technique that allows researchers to determine if there is elevated risk of chronic diseases 
among people with food insecurity independent of obesity. This is important because, as noted in the introduction, BMI is not always a reliable indicator of cardiometabolic risk; moreover, studies that rely on BMI as a proxy indicator of $\mathrm{CD}$ risk fail to include subgroups of people with FI who are not obese but may nevertheless be at increased risk of $C D$ because of the systemic harm created by the profoundly stressful experience of FI.

\subsection{Food Insecurity and Chronic Disease Associations Independent of Obesity}

The 15 studies included in the literature review that controlled for BMI in their analyses consistently reported associations between FI and CD risk independent of obesity. Seligman et al. [48], for instance, found that in a nationally representative sample of Americans, food insecurity was independently associated with diabetes. Although obesity among women was also associated with mild food insecurity, obesity accounted for only $20 \%$ of the increased odds of diabetes. Importantly, these studies indicate that a significant portion of the FI-CD relationship cannot be explained by obesity. We therefore urge researchers to include direct measures of CD risk biomarkers (such as high-sensitivity C-reactive protein assay for cardiometabolic risk, or lung function for asthma severity) along with BMI. Moreover, we underscore the need for biopsychosocial studies integrating qualitative work, biomarkers for $\mathrm{CD}$ and risk status, and standardized measures of FI to identify some of the apparent sources of association between FI and CD independent of obesity. This appears to be important both for diet-related and non-diet-related CD; among the studies demonstrating relationships between $\mathrm{CD}$ and food insecurity independent of obesity were both those focusing on diet-related diseases such as type 2 diabetes, and those focusing on non-diet-related diseases such as asthma.

These 15 studies are in the minority; little of the FI-CD research is designed to include statistical tests of the presence or absence of a mediating role for obesity in the FI-CD association. To correct for this, we recommend that even if researchers adopt more direct measures of cardiometabolic risk in their studies, they still include BMI as a control variable to assess the portion of the FI-CD relationship not accounted for by BMI.

\subsection{Possible Mechanisms of the FI-CD Association}

Many studies documented a FI-CD relationship without speculating about its causes, and those that did so almost universally addressed causes as theoretical possibilities rather than performing statistical tests to identify potential mediators. As a whole, the studies suggested three main categories of mediators. Each is summarized below.

The first, and most common, pathway that researchers proposed to link FI and $\mathrm{CD}$ is a broad category that we refer to as "economic mechanisms". In households with economic constraints, FI is more likely, as are a suite of other stressors that may interact cyclically with health to produce CD risk even when 
obesity is not present. As Crews et al. [38] and Tarasuk et al. [51] noted, unemployment related to chronic illness disability may be an important economic driver the CD-FI association. In such cases, the high cost of CD medications may leave insufficient money for nutritious food, and a lack of nutritious food exacerbates many CDs even in the absence of obesity, leading to higher treatment costs, medication nonadherence, and increased FI, in a cyclical relationship [36] [37]. Some of the work included here demonstrates that poverty leads to both FI and reduced healthcare usage (including medication nonadherence) [52] [53].

The six studies that included qualitative data also underscored the relevance of economic mechanisms linking FI and CDs. These studies demonstrated that low-income people with type 2 diabetes struggled to adhere to diabetic diets because of economic constraints including limited household finances, geographic unavailability of healthy foods, and lack of control over their own diets, rather than because of lack of awareness about the importance of dietary control [54] [55] [56]. These studies particularly highlighted the diabetes-inappropriate food available at food banks and the fact that dependence on food aid constrains one's ability to choose what to eat [32].

The wide range of pathways falling under the economic explanation attests to a social reality: that food insecurity rarely occurs in isolation; rather, it is only one of many physical, mental, and social health problems that households face when dealing with economic constraints. The studies that assessed psychological, social, and health outcomes found that FI is only one of many variables associated with CD risk among marginalized groups; other important CD predictors were depression and poor social support; culturally specific distress, gastrointestinal infection, low maternal education, and child learning disorders; and homelessness, stressful life events, low birthweight, and parental anxiety [34] [57] [58]. Food insecurity is both an index and an engine of poverty, and poverty is one of the most systematically health-harming forces to which humans can be exposed [59].

A second pathway potentially linking FI and CD in the absence of obesity is cyclically dysregulated eating patterns among FI individuals, such as reduced intake during food shortage followed by overconsumption when food is available, which leads to metabolic disruption that can lead to metabolic diseases even when a person is not obese [28] [40] [48] [60]. The eating disorder literature has long demonstrated that cyclical food restriction and bingeing interferes with normal metabolism via dysregulation of the HPA axis, which in turn appears to be related to visceral fat deposition (a strong risk factor for many chronic diseases) [61] [62] [63]. There is a parallel body of literature suggesting direct relationships between FI and dysregulated eating patterns [64] [65]. Food assistance programs might play a role in dysregulated eating because they emphasize processed foods and typically disburse benefits once monthly, thus leading to monthly cycles of feast-and-famine [1] [4] [66] [67]. Dysregulated eating behaviors-potentially perpetuated by reliance on once-monthly food aid or food 
banks-may predispose individuals to chronic diseases via metabolic dysregulation without the necessary precondition of obesity.

A third possible pathway linking FI and CD in the absence of obesity is high exposure to environmental toxins among people suffering from FI. Sattler and Bhargava [33], for instance, note the greater likelihood of smoking in low-income households as a potential risk factor for CDs independent of obesity. Along the same lines, Shiue [68] points out that people suffering from FI are also more likely to be exposed to high levels of environmental contaminants known to induce chronic diseases, such as bisphenol-a (BPA; an endocrine disrupter), pesticides (also endocrine disrupters), and antimony (a chemical element linked to liver dysfunction).

Strikingly few of the studies included in this review discussed biosocial pathways that might connect FI and CDs, such as the psychoneuroimmunological connections between distress and chronic health problems. Mangini et al. [45] make a nod to the whole-person approach to understanding FI-CD risk, pointing out that the associations between household poverty and child asthma must be understood within the framework of a life course model of health disparities. Pérez-Escamilla et al. [47] similarly point out that the chronic stress of FI could lead to higher stress hormones such as cortisol, which promotes abdominal fat storage, leading to type 2 diabetes and hypertension. None of these studies, however, considers the possibility that stress activation could lead to these CDs independently of the mechanism of abdominal fat storage-for instance, through generalized inflammation, which has been shown to be a strong predictor of chronic disease even when controlling for socioeconomic and dietary variables [69].

\subsection{Gaps in the Literature}

The literature was generally biased toward cross-sectional, quantitative studies based in North America. More longitudinal and qualitative studies are needed, as are studies conducted in low- and middle-income countries. Several studies emphasized the unique challenges faced by women, but work is needed on the qualitative and quantitative associations between FI and CD in men specifically, since men may experience FI and CD differently than women both physiologically and psychologically. Moreover, because studies addressing asthma and dyslipidemia were, as a whole, inconclusive about their relationships with FI, more work is needed on these potential relationships. No studies addressing chronic fatigue syndrome, fibromyalgia, lupus, or COPD were found during the review process. This may seem logical, given the widespread assumption that non-lifestyle-related diseases are less likely to be affected by food insecurity than are those directly related to diet or lifestyle. However, studies demonstrating clear links between food insecurity, stress response, and body inflammation suggest, at very least, that there is a potential link between non-diet-related diseases and food insecurity [69]. This warrants further exploration. 
Further study is also needed to determine if there are differences in the FI-CD relationship in low- and middle-income settings as opposed to industrialized countries; the three studies in Malaysia documented no association between FI and $\mathrm{CD}$, suggesting that this relationship may work differently in less-developed economies. Likewise, further work on the role of gender, minority race/ethnicity, and age with relevant comparison groups would help elucidate how these demographic characteristics may shape the FI-CD relationship.

Finally, the large proportion of studies publishing positive results could be of concern. While it is entirely plausible that this reflects a secular association between $\mathrm{CD}$ and FI, it could also reflect publication bias toward positive study results. We therefore encourage researchers who find no positive association between $\mathrm{CD}$ and FI to seek publication.

\subsection{Limitations}

This review was not designed to include the literature on the associations between health behaviors and food insecurity, which is substantial. The review also did not include studies conducted among populations with HIV/AIDS, which under treatment with regular antiretroviral drugs becomes very much like a chronic disease. There is significant literature, however, exploring relationships between FI and HIV/AIDS.

\section{Conclusions}

Food insecurity (FI) and chronic diseases (CD) are often related, as this literature review demonstrated. Moreover, there is evidence to suggest that FI and CD may be associated with one another independent of obesity, which is often assumed to be the variable mediating this association. Based on the literature reviewed here, other possible mediators of the FI-CD relationship include the effects of limited finances on health behaviors and treatment adherence (and vice-versa), micronutrient deficiencies that might lead to CDs in the absence of obesity, and exposure to environmental toxins leading to CDs. Few studies considered the potential direct effects of psychosocial stress resulting from systematic social and economic exclusion on CD risk, of which FI may be just one manifestation.

Because the evidence is limited, the present study concludes that more research is needed on the possible direct pathways linking FI and CDs without the reliance on obesity as the key explanatory variable mediating this relationship. Moreover, inclusion of mixed-method and longitudinal studies would be useful to help identify the mechanisms implicated in FI-CD relationships. Studies in low- and middle-income countries and studies that deal with non-diet-related CDs such as asthma, lupus, and fibromyalgia are also needed to correct for gaps in the literature. We caution researchers to avoid relying on BMI as a proxy for chronic disease risk or as an explanatory variable in studies of the FI-CD relationship, but instead to employ direct measures of $\mathrm{CD}$ risk and use BMI as a 
control variable. Finally, we encourage those finding no positive relationship between FI and CD to seek publication of their results in order to correct for potential publication bias.

\section{Acknowledgements}

None to report.

\section{Funding}

The second author's involvement in the research was supported by a stipend from the University of Alabama College of Arts and Sciences' Undergraduate Creativity and Research Academy.

\section{Conflict of Interest}

None declared.

\section{Consent for Publication}

Weaver and Fasel give consent for the manuscript in its present form to be reviewed for publication.

\section{Ethical Approval}

Since this was a systematic literature review, no ethical approval was required.

\section{References}

[1] Ashe, L.M. and Sonnino, R. (2013) At the Crossroads: New Paradigms of Food Security, Public Health Nutrition and School Food. Public Health Nutrition, 16, 1020-1027. https://doi.org/10.1017/S1368980012004326

[2] Dinour, L.M., Bergen, D. and Yeh, M. (2007) The Food Insecurity-Obesity Paradox: A Review of the Literature and the Role Food Stamps May Play. Journal of the American Dietetic Association, 107, 1952-1961.

[3] Finney Rutten, L.J., Yaroch, A.L., Colón-Ramos, U., Askew, W.J. and Story, M. (2010) Poverty, Food Insecurity, and Obesity: A Conceptual Framework for Research, Practice, and Policy. Journal of Hunger \& Environmental Nutrition, 5, 403-415.

[4] Franklin, B., Jones, A., Love, D., Puckett, S., Macklin, J. and White-Means, S. (2012) Exploring Mediators of Food Insecurity and Obesity: A Review of Recent Literature. Journal of Community Health, 37, 253-264. https://doi.org/10.1007/s10900-011-9420-4

[5] Ivers, L.C. and Cullen, K.A. (2011) Food Insecurity: Special Considerations for Women. The American Journal of Clinical Nutrition, 94, 1740S-1744S.

[6] Ramsey, R., Giskes, K., Turrell, G. and Gallegos, D. (2012) Food Insecurity among Adults Residing in Disadvantaged Urban Areas: Potential Health and Dietary Consequences. Public Health Nutrition, 15, 227-237. https://doi.org/10.1017/S1368980011001996

[7] Holben, D.H. and Pheley, A.M. (2006) Diabetes Risk and Obesity in Food-Insecure Households in Rural Appalachian Ohio. Preventing Chronic Disease, 3, A82. 
[8] Holben, D. (2010) Position of the American Dietetic Association: Food Insecurity in the United States. Journal of the American Dietetic Association, 110, 1368-1377.

[9] Castillo, D.C., Ramsey, N.L.M., Yu, S.S.K., Ricks, M., Courville, A.B. and Sumner, A.E. (2012) Inconsistent Access to Food and Cardiometabolic Disease: The Effect of Food Insecurity. Current Cardiovascular Risk Reports, 6, 245-250.

https://doi.org/10.1007/s12170-012-0236-2

[10] Dyson, P. (2012) Food Insecurity: What Does It Mean to People with Diabetes? Practical Diabetes, 29, 89a-90a.

[11] Gucciardi, E., Vahabi, M., Norris, N., Del Monte, J.P. and Farnum, C. (2014) The Intersection between Food Insecurity and Diabetes: A Review. Current Nutrition Reports, 3, 324-332. https://doi.org/10.1007/s13668-014-0104-4

[12] Hadley, C. and Crooks, D.L. (2012) Coping and the Biosocial Consequences of Food Insecurity in the 21st Century. American Journal of Physical Anthropology, 149, S72-S94.

[13] Laraia, B.A. (2013) Food Insecurity and Chronic Disease. Advances in Nutrition, 4, 203-212.

[14] Lee, J.S., Gundersen, C., Cook, J., Laraia, B. and Johnson, M.A. (2012) Food Insecurity and Health across the Lifespan. Advances in Nutrition, 3, 744-745.

[15] Kurpad, A.V., Varadharajan, K.S. and Aeberli, I. (2011) The Thin-Fat Phenotype and Global Metabolic Disease Risk. Current Opinion in Clinical Nutrition \& Metabolic Care, 14, 542-547. https://doi.org/10.1097/MCO.0b013e32834b6e5e

[16] Yajnik, C.S., Fall, C.H.D., Coyaji, K.J., Hirve, S.S., Rao, S., Barker, D.J.P., Joglekar, C. and Kellingray, S. (2003) Neonatal Anthropometry: The Thin-Fat Indian Baby: The Pune Maternal Nutrition Study. International Journal of Obesity, 27, 173-180. https://doi.org/10.1038/sj.ijo.802219

[17] Barker, D.J.P. (2015) Fetal Origins of Coronary Heart Disease. BMJ, 311, 171-174. https://doi.org/10.1136/bmj.311.6998.171

[18] Clausen, T.D., Mathiesen, E.R., Hansen, T., Pederson, O., Jensen, D.M., Lauenburg, J., et al. (2008) High Prevalence of Type 2 Diabetes and Pre-Diabetes in Adult Offspring of Women with Gestational Diabetes Mellitus or Type 1 Diabetes: The Role of Intrauterine Hyperglycemia. Diabetes Care, 31, 340-346. https://doi.org/10.2337/dc07-1596

[19] Roseboom, T., de Rooij, S. and Painter, R. (2006) The Dutch Famine and Its Long-Term Consequences for Adult Health. Early Human Development, 82, 485-491. https://doi.org/10.1016/j.earlhumdev.2006.07.001

[20] Schulz, L.C. (2010) The Dutch Hunger Winter and the Developmental Origins of Health and Disease. Proceedings of the National Academy of Sciences of the United States of America, 107, 16757-16758. https://doi.org/10.1073/pnas.1012911107

[21] Thurner, S., Klimek, P., Szell, M., Duftschmid, G., Endel, G., Willer, A.K. and Kasper, D.C. (2013) Quantification of Excess Risk for Diabetes for those Born in Times of Hunger, in an Entire Population of a Nation, across a Century. Proceedings of the National Academy of Sciences of the United States of America, 110, 4703-4707. https://doi.org/10.1073/pnas.1215626110

[22] Hruschka, D.J. and Hadley, C. (2016) How Much Do Universal Anthropometric Standards Bias the Global Monitoring of Obesity and Undernutrition? Obesity Reviews, 17, 1030-1039.

[23] Lyons, A.A., Park, J. and Nelson, C.H. (2008) Food Insecurity and Obesity: A Comparison of Self-Reported and Measured Height and Weight. American Journal of Public Health, 98, 751-757. 
[24] Weaver, L.J. and Hadley, C. (2009) Moving Beyond Hunger and Nutrition: A Systematic Review of the Evidence Linking Food Insecurity and Mental Health in Developing Countries. Ecology of Food and Nutrition, 48, 263-284.

[25] Theall, K.P., Dunaway, L.F. and Mabile, E. (2013) Food Security and C-Reactive Protein in Adolescents. American Journal of Public Health, 103, e5.

[26] Mohamadpour, M., Sharif, Z.M. and Keysami, M.A. (2012) Food Insecurity, Health and Nutritional Status among Sample of Palm-Plantation Households in Malaysia. Journal of Health, Population and Nutrition, 30, 291-302. https://doi.org/10.3329/jhpn.v30i3.12292

[27] Nur Atiqah, A., Norazmir, M.N., Khairil Anuar, M.I., Mohd Fahmi, M. and Norazlanshah, H. (2015) Food Security Status: It's Association with Inflammatory Marker and Lipid Profile among Young Adult. International Food Research Journal, 22, $1855-1863$.

[28] Shariff, Z.M., Sulaiman, N., Jalil, R.A., Yen, W.C., Yaw, Y.H., Taib, M.N.M., Kandiah, M. and Lin, K.G. (2014) Food Insecurity and the Metabolic Syndrome among Women from Low Income Communities in Malaysia. Asia Pacific Journal of Clinical Nutrition, 23, 138-147.

[29] Asaad, G. and Chan, C.B. (2012) Relationship of Diet Quality to Food Security and Nutrition Knowledge in Low-Income, Community-Dwelling Elders with Type 2 Diabetes Mellitus: A Pilot Study. Canadian Journal of Diabetes, 36, 310-313.

[30] Hendrickson, M.A., O’Riordan, M.A., Arpilleda, J.C. and Heneghan, A.M. (2010) Effects of Food Insecurity on Asthma Outcomes in the Pediatric Emergency Department. Pediatric Emergency Care, 26, 823-829. https://doi.org/10.1097/PEC.0b013e3181fb0dab

[31] Homenko, D.R., Morin, P.C., Eimicke, J.P., Teresi, J.A. and Weinstock, R.S. (2010) Food Insecurity and Food Choices in Rural Older Adults with Diabetes Receiving Nutrition Education via Telemedicine. Journal of Nutrition Education and Behavior, 42, 404-409.

[32] Hwang, S.W. and Bugeja, A.L. (2000) Barriers to Appropriate Diabetes Management among Homeless People in Toronto. Canadian Medical Association Journal, 163, 161-165.

[33] Sattler, E.L. and Bhargava, V. (2016) Food Insecurity and Cardiovascular Risk factors in US Adolescents. Journal of Applied Research on Children, 6, 12.

[34] Weigel, M.M., Armijos, R.X., Hall, Y.P., Ramirez, Y. and Orozco, R. (2007) The Household Food Insecurity and Health Outcomes of US-Mexico Border Migrant and Seasonal Farmworkers. Journal of Immigrant and Minority Health, 9, 157-169. https://doi.org/10.1007/s10903-006-9026-6

[35] Tayie, F.A. and Zizza, C.A. (2009) Food Insecurity and Dyslipidemia among Adults in the United States. Preventive Medicine, 48, 480-485. https://doi.org/10.1016/j.ypmed.2009.03.003

[36] Bawadi, H.A., Ammari, F., Abu-Jamous, D., Khader, Y.S., Bataineh, S. and Tayyem, R.F. (2012) Food Insecurity Is Related to Glycemic Control Deterioration in Patients with Type 2 Diabetes. Clinical Nutrition, 31, 250-254.

[37] Berkowitz, S.A., Baggett, T.P., Wexler, D.J., Huskey, K.W. and Wee, C.C. (2013) Food Insecurity and Metabolic Control among U.S. Adults with Diabetes. Diabetes Care, 36, 3093-3099. https://doi.org/10.2337/dc13-0570

[38] Crews, D.C., Kuczmarski, M.F., Grubbs, V., Hedgeman, E., Shahinian, V.B., Evans, M.K., et al. (2014) Effect of Food Insecurity on Chronic Kidney Disease in Lower-Income Americans. American Journal of Nephrology, 39, 27-35. 
https://doi.org/10.1159/000357595

[39] de Cássia Ribeiro-Silva, R.C., Oliveira-Assis, A.M., Junqueira, S.B., Fiaccone, R.L., dos Santos, S.M.C., Barreto, M.L., et al. (2014) Food and Nutrition Insecurity: A Marker of Vulnerability to Asthma Symptoms. Public Health Nutrition, 17, 14-19. https://doi.org/10.1017/S1368980012005551

[40] Fitzgerald, N., Hromi-Fiedler, A., Segura-Pérez, S. and Pérez-Escamilla, R. (2011) Food Insecurity Is Related to Increased Risk of Type 2 Diabetes among Latinas. Ethnicity \& Disease, 21, 328-334.

[41] Hasan-Ghomi, M., Ejtahed, H.S., Mirmiran, P., Hosseini-Esfahani, F., Sarbazi, N., Azizi, F. and Sadeghian, S. (2015) Relationship of Food Security with Type 2 Diabetes and Its Risk Factors in Tehranian Adults. International Journal of Preventive Medicine, 6, 98. https://doi.org/10.4103/2008-7802.167086

[42] Heerman, W.J., Wallston, K.A., Osborn, C.Y., Bian, A., Schlundt, D.G., Barto, S.D. and Rothman, R.L. (2016) Food Insecurity Is Associated with Diabetes Self-Care Behaviours and Glycaemic Control. Diabetic Medicine, 33, 844-850

[43] Liu, J., Park, Y.M., Berkowitz, S.A., Hu, Q., Han, K., Ortaglia, A., et al. (2015) Gender Differences in the Association between Food Insecurity and Insulin Resistance among US Adults: National Health and Nutrition Examination Survey, 2005-2010. Annals of Epidemiology, 25, 643-648.

[44] Lyles, C.R., Wolf, M.S., Schillinger, D., Davis, T.C., Dewalt, D., Dahlke, A.R., et al. (2013) Food Insecurity in Relation to Changes in Hemoglobin $A_{1 c}$, Self-Efficacy, and Fruit/Vegetable Intake during a Diabetes Educational Intervention. Diabetes Care, 36, 1448-1453. https://doi.org/10.2337/dc12-1961

[45] Mangini, L.D., Hayward, M.D., Dong, Y.Q. and Forman, M.R. (2015) Household Food Insecurity Is Associated with Childhood Asthma. The Journal of Nutrition, $145,2756-2764$

[46] Mayer, V.L., McDonough, K., Seligman, H., Mitra, N. and Long, J.A. (2016) Food Insecurity, Coping Strategies and Glucose Control in Low-Income Patients with Diabetes. Public Health Nutrition, 19, 1103-1111. https://doi.org/10.1017/S1368980015002323

[47] Pérez-Escamilla, R., Villalpando, S., Shamah-Levy, T. and Humaran, I.M.G. (2014) Household Food Insecurity, Diabetes and Hypertension among Mexican Adults: Results from Ensanut 2012. Salud Pública de México, 56, 562-570.

[48] Seligman, H.K., Bindman, A.B., Vittinghoff, E., Kanaya, A.M. and Kushel, M.B. (2007) Food Insecurity Is Associated with Diabetes Mellitus: Results from the $\mathrm{Na}$ tional Health Examination and Nutrition Examination Survey (NHANES) 1999-2002. Journal of General Internal Medicine, 22, 1018-1023. https://doi.org/10.1007/s11606-007-0192-6

[49] Shin, J.I., Bautista, L.E., Walsh, M.C., Malecki, K.C. and Nieto, F.J. (2015) Food Insecurity and Dyslipidemia in a Representative Population-Based Sample in the US. Preventive Medicine, 77, 186-190. https://doi.org/10.1016/j.ypmed.2015.05.009

[50] Silverman, J., Krieger, J., Kiefer, M., Hebert, P., Robinson, J. and Nelson, K. (2015) The Relationship between Food Insecurity and Depression, Diabetes Distress and Medication Adherence among Low-Income Patients with Poorly-Controlled Diabetes. Journal of General Internal Medicine, 30, 1476-1480. https://doi.org/10.1007/s11606-015-3351-1

[51] Tarasuk, V., Mitchell, A., McLaren, L. and Lynn, M. (2013) Chronic Physical and Mental Health Conditions among Adults May Increase Vulnerability to Household Food Insecurity. The Journal of Nutrition, 143, 1785-1793. 
[52] Bhargava, V., Lee, J.S., Jain, R., Johnson, M.A. and Brown, A. (2012) Food Insecurity Is Negatively Associated with Home Health and Out-of-Pocket Expenditures in Older Adults. The Journal of Nutrition, 42, 1888-1895.

[53] Sattler, E.L. and Lee, J.S. (2013) Persistent Food insecurity Is Associated with Higher Levels of Cost-Related Medication Nonadherence in Low-Income Older Adults. Journal of Nutrition in Gerontology and Geriatrics, 32, 41-58.

[54] Chan, J., DeMelo, M., Gingras, J. and Gucciardi, E. (2015) Challenges of Diabetes Self-Management in Adults Affected by Food Insecurity in a Large Urban Centre of Ontario, Canada. International Journal of Endocrinology and Metabolism, 2015, Article ID: 903468.

[55] Chaufan, C., Davis, M. and Constantino, S. (2011) The Twin Epidemics of Poverty and Diabetes: Understanding Diabetes Disparities in a Low-Income Latino and Immigrant Neighborhood. Journal of Community Health, 36, 1032-1043. https://doi.org/10.1007/s10900-011-9406-2

[56] Cuesta-Briand, B., Saggers, S. and McManus, A. (2011) "You Get the Quickest and the Cheapest Stuff You Can": Food Security Issues among Low-Income Earners Living with Diabetes. Australasian Medical Journal, 4, 683-691.

[57] Vozoris, N.T. and Tarasuk, V.S. (2003) Household Food Insufficiency Is Associated with Poorer Health. The Journal of Nutrition, 133, 120-126.

[58] Weinreb, L., Wehler, C., Perloff, J., Scott, R., Hosmer, D., Sagor, L., et al. (2002) Hunger: Its Impact on Children's Health and Mental Health. Pediatrics, 110, e41. https://doi.org/10.1542/peds.110.4.e41

[59] Wilkinson, R. (2009) Unhealthy Societies: The Afflictions of Inequality. Rutledge, London.

[60] Galesloot, S., McIntyre, L., Fenton, T. and Tyminski, S. (2012) Food Insecurity in Canadian Adults Receiving Diabetes Care. Canadian Journal of Dietetic Practice and Research, 73, e261-e266.

[61] Bjorntorp, P. (2001) Do Stress Reactions Cause Abdominal Obesity and Comorbidities? Obesity Reviews, 2, 73-86.

[62] Licinio, J., Wong, M.L. and Gold, P.W. (1996) The Hypothalamic-Pituitary-Adrenal Axis in Anorexia Nervosa. Psychiatry Research, 62, 75-83.

[63] Rosmond, R., Dallman, M.F. and Bjöntorp, P. (1998) Stress-Related Cortisol Secretion in Men: Relationships with Abdominal Obesity and Endocrine, Metabolic and Hemodynamic Abnormalities. The Journal of Clinical Endocrinology \& Metabolism, 83, 1853-1859.

[64] Allen, M.W. and Wilson, M. (2005) Materialism and Food Security. Appetite, 45, 314-323. https://doi.org/10.1016/j.appet.2005.06.005

[65] Bruening, M., MacLehose, R., Loth, K., Story, M. and Sztainer, D.N. (2012) Feeding a Family in a Recession: Food Insecurity among Minnesota Parents. American Journal of Public Health, 102, 520-526.

[66] Correll, M. (2010) Getting Fat on Government Cheese: The Connection between Social Welfare Participation, Gender, and Obesity in America. Duke Journal of Gender Law \& Policy, 18, 45-77.

[67] Leung, C.W., Epel, E.S., Ritchie, L.D., Crawford, P.B. and Laraia, B.A. (2014) Food Insecurity Is Inversely Associated with Diet Quality of Lower-Income Adults. Journal of the Academy of Nutrition and Dietetics, 114, 1943-1953.

[68] Shiue, I. (2016) People with Diabetes, Respiratory, Liver or Mental Disorders, Higher Urinary Antimony, Bisphenol A, or Pesticides had Higher Food Insecurity: USA NHANES, 2005-2006. Environmental Science and Pollution Research, 23, 
198-205. https://doi.org/10.1007/s11356-015-5677-y

[69] Gowda, C., Hadley, C. and Aiello, A.E. (2012) The Association between Food Insecurity and Inflammation in the US Adult Population. American Journal of Public Health, 102, 1579-1586. https://doi.org/10.2105/AJPH.2011.300551 